\title{
TRANSFER MATRICES, NON-HERMITIAN HAMILTONIANS AND RESOLVENTS: SOME SPECTRAL IDENTITIES
}

\author{
Luca Molinari \\ Dipartimento di Fisica dell'Università di Milano and I.N.F.N. \\ Via Celoria 16, 20133 Milano
}

\begin{abstract}
I consider the $N$-step transfer matrix $T$ for a general block Hamiltonian, with eigenvalue equation

$$
L_{n} \psi_{n+1}+H_{n} \psi_{n}+L_{n-1}^{\dagger} \psi_{n-1}=E \psi_{n}
$$

where $H_{n}$ and $L_{n}$ are matrices, and provide its explicit representation in terms of blocks of the resolvent of the Hamiltonian matrix for the system of length $N$ with boundary conditions $\psi_{0}=\psi_{N+1}=0$. I then introduce the related Hamiltonian for the case $\psi_{0}=$ $z^{-1} \psi_{N}$ and $\psi_{N+1}=z \psi_{1}$, and provide an exact relation between the trace of its resolvent and $\operatorname{Tr}(T-z)^{-1}$, together with an identity of Thouless type connecting $\operatorname{Tr}(\log |T|)$ with the Hamiltonian eigenvalues for $z=e^{i \phi}$. The results are then extended to $T^{\dagger} T$ by showing that it is itself a transfer matrix. Besides their own mathematical interest, the identities should be useful for an analytical approach in the study of spectral properties of a physically relevant class of transfer matrices.

P.A.C.S.: 02.10.Sp (theory of matrices), 05.60 (theory of quantum transport), 71.23 (Anderson model), 72.17.Rn (Quantum localization)
\end{abstract}

To appear in October 1998, on J. Phys. A: Math. Gen.

E-Mail address: luca.molinari@milano.infn.it 


\section{$\S 1$ Introduction.}

Several discretized models are described by a Hamiltonian matrix $\mathcal{H}$ with tridiagonal structure made of blocks $H_{n}=H_{n}^{\dagger}$ along the main diagonal, and blocks $L_{n}$, $L_{n}^{\dagger}$, with $\operatorname{det} L_{n} \neq 0$, respectively in the adjacent upper and lower diagonals, the blocks having size $M \times M$. The diagonal matrices may describe the inner dynamics of a sequence of finite subsystems, and the off-diagonal matrices are the couplings among neighbouring ones.

An important and extensively studied example is Anderson's model for electronic transport in a $\mathrm{D}$-dimensional lattice with random potential, which for $\mathrm{D}=3$ exhibits a metal-insulator transition [1]. The single matrices $H_{n}$ are random Hamiltonians for the isolated slices of dimension D-1 and, in the simplest case, the couplings $L_{n}$ are proportional to the unit matrix, as implied by the discretization of the Laplacian. They may also be complex, in the presence of a magnetic field [2], or random, due to random hopping amplitudes [3]. Another frequently studied model is the ensemble of band random matrices [4], where $H_{n}$ is a member of GOE or GUE and $L_{n}$ are random and lower triangular matrices. They found applications in quantum chaos [5], one particle mesoscopic transport [6] and the propagation of two particles in disordered media [7]. The block structure also arises in the Fourier representation of the Floquet Hamiltonian $H_{t}=H_{0}+V^{\dagger} e^{i \omega t}+V e^{-i \omega t}-i \partial_{t}$, giving $H_{n}=H_{0}+n \omega$ and $L=V$. Block Hamiltonians have also been investigated in the context of matrix models [8], the blocks being rotationally invariant for the methods to apply.

The eigenvalue equation for $\mathcal{H}$, in block components, is:

$$
L_{n} \psi_{n+1}+H_{n} \psi_{n}+L_{n-1}^{\dagger} \psi_{n-1}=E \psi_{n}
$$

One is often interested in asymptotic properties of eigenvectors. A basic tool for this analysis, which exploits the recursive content of the eigenvalue equation, is the transfer matrix, connecting pairs of components of the vector

$$
\left(\begin{array}{c}
L_{N} \psi_{N+1} \\
\psi_{N}
\end{array}\right)=T(E)\left(\begin{array}{c}
\psi_{1} \\
L_{0}^{\dagger} \psi_{0}
\end{array}\right)
$$

The transfer matrix has size $2 M \times 2 M$ and it is convenient to factorize it as follows:

$$
\begin{gathered}
T(E)=T_{N}(E) \Sigma_{N-1} T_{N-1}(E) \Sigma_{N-2} \ldots \Sigma_{1} T_{1}(E) \\
T_{k}(E)=\left(\begin{array}{cc}
E-H_{k} & -I \\
I & 0
\end{array}\right), \quad \Sigma_{k}=\left(\begin{array}{cc}
L_{k}^{-1} & 0 \\
0 & L_{k}^{\dagger}
\end{array}\right)
\end{gathered}
$$

By allowing for complex values of the parameter $E$, for the single factors and then for the whole product, one obtains the important symplectic property

$$
T\left(E^{*}\right)^{\dagger} \sigma_{2} T(E)=\sigma_{2}, \quad \sigma_{2}=\left(\begin{array}{cc}
0 & -I \\
I & 0
\end{array}\right)
$$

where $I$ is the unit matrix of size $M$. Another consequence of the factorized expression is $\operatorname{det} T(E)=\prod_{k} \operatorname{det}\left[L_{k}^{\dagger} L_{k}^{-1}\right]$, that implies $|\operatorname{det} T(E)|=1$. 
General theorems assert that the eigenvalues $t_{a}$ of transfer matrices built with random factors grow or decrease exponentially with $N[9]$, allowing the definition of characteristic exponents

$$
\gamma_{a}(E)=\lim _{N \rightarrow \infty} \frac{1}{N} \log \left|t_{a}(E)\right|
$$

which, in the case of $\left(T^{\dagger} T\right)^{1 / 2}$, constitute the Lyapunov spectrum of the model. The analytical derivation of a Lyapunov spectrum is usually extremely difficult, the alternative being a careful numerical work to cope with exponential instabilities. For $2 \times 2$ matrices $(M=1)$, a relevant formula by Herbert and Jones, rediscussed by Thouless and bearing his name, connects the single Lyapunov exponent to the eigenvalue density of the ensemble of Jacobi Hamiltonians [10]. However, the density is by no means a simpler problem; an exception is Lloyd's model, characterized by diagonal disorder with Cauchy distribution: in this case the analytical expression of $\gamma(E)$ is known [10]. The statistical properties of the Lyapunov exponent and various generalizations have been investigated extensively by Pendry [11].

One of the rare solvable examples in more than one dimension is in the work [12] by Isopi and Newman, who studied products of matrices all of whose entries are identically distributed random variables, and found analytically a "triangle law" for the Lyapunov spectrum; Cook and Derrida considered the case of randomly sparse matrices [13]. A beautiful statistical theory of transfer matrices, based on few physical contraints, has been introduced by Mello and others to describe transport properties in disordered multichannel conductors, obtaining the observed value for universal conductance fluctuations [14]. In general, however, the transfer matrix is a derivated object, which inherits a structure from the specific recurrence or dynamical equation under examination. Its statistical properties depend in a complicated way on fluctuating parameters that enter more naturally, for example, in the characterization of an ensemble of Hamiltonians.

For the class of Hamiltonians we are considering, analytical results are lacking. Many extensive numerical calculations have been carried out for the Anderson model. It was by means of transfer matrices that Kramer and MacKinnon first exhibited the metal-insulator transition in 3 dimensions [15], through the different scaling behaviour in the transverse area $M$ of the smallest Lyapunov exponent. A recent numerical study by Markos [16] provides the whole Lyapunov spectrum, which is sensitive to the transition. The Lyapunov spectrum of band random matrices was obtained numerically by Kottos et al. [17], with a discussion of finite size corrections.

The aim of this paper is to investigate some general mathematical properties of the transfer matrix $T(E)$ that arise from the block structure of a single but generic Hamiltonian matrix $\mathcal{H}$, of size $N M$, corresponding to (1.1) with boundary condition $\psi_{0}=\psi_{N+1}=0$.

In [18] I showed that the eigenvalues of the transfer matrix are most directly related to those of a matrix $\mathcal{H}(z)$ of size $N M$, which in general is not Hermitian and has block 
structure

$$
\mathcal{H}(z)=\left(\begin{array}{cccccc}
H_{1} & L_{1} & & & & \frac{1}{z} I \\
L_{1}^{\dagger} & H_{2} & L_{2} & & & \\
& L_{2}^{\dagger} & \ldots & & & \\
& & & \ldots & H_{N-1} & L_{N-1} \\
z I & & & & L_{N-1}^{\dagger} & H_{N}
\end{array}\right)
$$

resulting from the eigenvalue equation (1.1) with boundary conditions specified through a complex parameter $z$ :

$$
L_{N} \psi_{N+1}=z \psi_{1} \quad L_{0}^{\dagger} \psi_{0}=\frac{1}{z} \psi_{N}
$$

The relation is based on the simple statement, whose proof is straightforward:

$A$ vector $\left(\psi_{1}, \psi_{2}, \ldots, \psi_{N}\right)$ is an eigenvector of $\mathcal{H}(z)$ with eigenvalue $E$ if and only if $\left(z \psi_{1}, \psi_{N}\right)$ is an eigenvector of $T(E)$ with eigenvalue $z$, the components $\psi_{2} \ldots \psi_{N-1}$ being linked to $\psi_{N}$ and $L_{N} \psi_{N+1}=z \psi_{1}$ by (1.1).

It implies that the characteristic polynomials of $T(E)$ and $\mathcal{H}(z)$ are proportional, and eventually leads to the following "duality relation":

$$
\operatorname{det}[T(E)-z]=(-z)^{M} \operatorname{det}\left(L_{N-1} \ldots L_{1}\right)^{-1} \operatorname{det}[E-\mathcal{H}(z)]
$$

A new proof will be given in $\S 2$, after having derived an explicit representation of the matrix $T(E)$ in terms of the corner blocks $G_{i j}, i, j=1, N$, of the resolvent $G=(\mathcal{H}-E)^{-1}$ of the Hamiltonian matrix $\mathcal{H}$, for the system of length $N$. The Hamiltonian has block structure (1.6) with null matrices replacing the $z$-dependent corner blocks, corresponding to the boundary requirement $\psi_{0}=\psi_{N+1}=0$.

In this paper it is noted that the derivative in the variable $z$ gives a relation between resolvents

$$
\operatorname{Tr}\left(\frac{1}{T(E)-z}\right)=-\frac{M}{z}+\frac{\partial}{\partial z} \log \operatorname{det}[E-\mathcal{H}(z)]
$$

The equation also follows from a representation of $[T(E)-z]^{-1}$ in terms of the corner blocks of the resolvent $\tilde{G}=(\mathcal{H}(z)-E)^{-1}$, to be obtained in $\S 4$. Did $\mathcal{H}$ belong to an ensemble, it would provide access to the spectral density of $T(E)$ by relating the difficult problem of averaging the resolvent of the transfer matrix, which depends on the ensemble parameters in a complex way, to the average of a ratio of determinants of the Hamiltonian itself.

The general discussion for $T(E)$ will be extended in $\S 5$ to cover the relevant matrix $T(E)^{\dagger} T(E)$, by showing that it is itself the transfer matrix of a tridiagonal block Hamiltonian, of size $2 N M$.

The main results of the paper can be summarized in the following equations: relation (1.9) or its equivalent (3.6) among traces of resolvents, (2.3) and (4.3) that provide the representation of the transfer matrix in terms of corner blocks of resolvents, (3.5) and (5.4) that generalize Thouless' relation to $M>1$ and relate Lyapunov exponents to the eigenvalues of the Hamiltonian matrix, the duality relation (5.6) for ther matrix $T^{\dagger} T$.

The described results are exact and general. Hopefully, this work should provide an analytical framework for the hard task of investigating average spectral properties of transfer 
matrices, constructed from an ensemble of Hamiltonians of this type. One more motivation is the recent interest in Non-Hermitian matrices, which are now extensively investigated mainly in the one-dimensional case $M=1$ [19], precisely with the tridiagonal structure and boundary conditions that are here generalized. In one dimension, the eigenvalues are distributed along curves in the complex plane [20] and real eigenvalues correspond to delocalized eigenvectors [21]. The here established relationship with the transfer matrix makes non Hermitian matrices an obvious object for investigating localization properties of eigenvectors. For $M=1$ this was done by Brouwer et al. [22].

\section{$\S 2$ The transfer matrix and the resolvent of $\mathcal{H}$.}

In this section I obtain a block representation of $T(E)$ in terms of the corner blocks of the resolvent of $\mathcal{H}$. I then prove the duality relation (1.8).

For complex $E$, let us introduce the resolvent $G(E)=(\mathcal{H}-E)^{-1}$. It is a matrix made of $N^{2}$ square blocks $G_{i j}$ of size $M \times M$. The relation $\left[G(E)_{i j}\right]^{\dagger}=G\left(E^{*}\right)_{j i}$ holds. By definition:

$$
L_{i-1}^{\dagger} G_{i-1, j}+\left(H_{i}-E\right) G_{i, j}+L_{i} G_{i+1, j}=I \delta_{i j}
$$

By solving the recurrence relations for $j=1$ and $j=N$, one obtains two identities involving the transfer matrix

$$
\left(\begin{array}{c}
0 \\
G_{N, 1}
\end{array}\right)=T(E)\left(\begin{array}{c}
G_{1,1} \\
-I
\end{array}\right), \quad\left(\begin{array}{c}
-I \\
G_{N, N}
\end{array}\right)=T(E)\left(\begin{array}{c}
G_{1, N} \\
0
\end{array}\right)
$$

They can be joined into a matrix relation, which gives a representation of the transfer matrix in terms of the corner blocks of the resolvent:

$$
\begin{aligned}
T(E) & =\left(\begin{array}{cc}
-I & 0 \\
G_{N, N} & G_{N, 1}
\end{array}\right)\left(\begin{array}{cc}
G_{1, N} & G_{1,1} \\
0 & -I
\end{array}\right)^{-1}= \\
& =\left(\begin{array}{cc}
-G_{1, N}^{-1} & -G_{1, N}^{-1} G_{1,1} \\
G_{N, N} G_{1, N}^{-1} & -G_{N, 1}+G_{N, N} G_{1, N}^{-1} G_{1,1}
\end{array}\right)
\end{aligned}
$$

One checks that the symplectic property (1.4) is identically satisfied. Note that each block component of $T(E)$ is a matrix polynomial in $E$, and is here explicitly represented in terms of the resolvent of the Hamiltonian. By varying the number $N$ of factors in $T(E)$, one obtains a collection of matrix polynomials which follow orthogonality relations that extend to $M>1$ the familiar notion of othogonal polynomials of Jacobi matrices [23].

To the end of deriving the duality relation, I first prove

$$
\operatorname{det} G_{1, N}^{-1}=\operatorname{det}\left[L_{1} \ldots L_{N-1}\right]^{-1} \operatorname{det}[E-\mathcal{H}]
$$

Proof: a vector $\left(\psi_{1}, \ldots, \psi_{N}\right)$ is an eigenvector of $\mathcal{H}$ with eigenvalue $E$ if and only if it solves (1.1) with boundary conditions $\psi_{N+1}=\psi_{0}=0$. These conditions, by eqs. (1.2) and (2.3b), are equivalent to the requirement $0=G_{1, N}^{-1} \psi_{1}$. By construction $T(E)$ is a polynomial in $E$ of degree $N$ with matrix coefficients; then $\operatorname{det} G_{1, N}^{-1}$ is a polynomial in $E$ of degree $N M$ and leading term $(-1)^{M} E^{M N} \operatorname{det}\left[L_{N-1}^{-1} \ldots L_{1}^{-1}\right]$. The polynomials $\operatorname{det}[E-\mathcal{H}]$ and 
$\operatorname{det}\left[G_{1, N}^{-1}\right]$ share all zeros and are therefore proportional by a numerical factor obtained from comparison of the leading terms.

I now give a proof of the duality relation (1.8), alternative to that provided in [18].

Proof: by writing $\mathcal{H}(z)=\mathcal{H}+V(z)$, where $V(z)$ is zero everywhere except for the corner blocks $V_{1, N}=\frac{1}{z} I$ and $V_{N, 1}=z I$, one calculates:

$$
\begin{aligned}
\operatorname{det}[E-\mathcal{H}(z)] & =\operatorname{det}[E-\mathcal{H}] \operatorname{det}[I+G(E) V(z)]= \\
& =\operatorname{det}[E-\mathcal{H}] \operatorname{det}\left(\begin{array}{cc}
I+z G_{1, N} & \frac{1}{z} G_{1,1} \\
z G_{N, N} & I+\frac{1}{z} G_{N, 1}
\end{array}\right)
\end{aligned}
$$

On the other hand, by using algebraic properties of determinants, one obtains from eq. $(2.3 \mathrm{~b})$ :

$$
\operatorname{det}[T(E)-z]=z^{M} \operatorname{det}\left[G_{1, N}^{-1}\right] \operatorname{det}\left(\begin{array}{cc}
I+z G_{1, N} & \frac{1}{z} G_{1,1} \\
z G_{N, N} & I+\frac{1}{z} G_{N, 1}
\end{array}\right)
$$

By taking into account property (2.4), the duality relation (1.8) follows.

\section{$\S 3$ The duality relations.}

I here discuss some consequences of the duality relation,

$$
\operatorname{det}[T(E)-z]=(-z)^{M} \operatorname{det}\left(L_{N-1} \ldots L_{1}\right)^{-1} \operatorname{det}[E-\mathcal{H}(z)]
$$

An identity for the inverse transfer matrix is obtained from the adjoint of (3.1), with the aid of the properties $T\left(E^{*}\right)^{\dagger}=-\sigma_{2} T(E)^{-1} \sigma_{2}$ and $\mathcal{H}\left(z^{*}\right)^{\dagger}=\mathcal{H}(1 / z)$ :

$$
\operatorname{det}\left[T(E)^{-1}-z\right]=(-z)^{M} \operatorname{det}\left(L_{N-1}^{\dagger} \ldots L_{1}^{\dagger}\right)^{-1} \operatorname{det}[E-\mathcal{H}(1 / z)] .
$$

The product of the two identities immediately yields an identity which was used in [18] to study the structure of bands and the dynamics of eigenvalues of the Hermitian Hamiltonians $\mathcal{H}\left(e^{i \varphi}\right)$ :

$$
\operatorname{det}\left[T(E)+T(E)^{-1}-\left(z+\frac{1}{z}\right)\right]=\left|\operatorname{det}\left(L_{N-1} \ldots L_{1}\right)\right|^{-2} \operatorname{det}[E-\mathcal{H}(z)] \operatorname{det}[E-\mathcal{H}(1 / z)]
$$

A simple general consequence of (3.1) is that, for $\operatorname{Im} E \neq 0$, the transfer matrix $T(E)$ has no eigenvalues on the unit circle, since the right term of (3.1) never vanishes for a Hermitian matrix. More generally, this is true for $E$ not in the union of the bands $B_{k}, k=1 \ldots N M$, each one being defined as the interval of the real axis spanned by the eigenvalue $E_{k}(\varphi)$ of the Hermitian matrix $\mathcal{H}\left(e^{i \phi}\right)$, as $\varphi$ varies in $[0,2 \pi)$.

From the symplectic property (1.4) it follows that if $t$ is an eigenvalue of $T(E)$ with $|t| \neq 1$, then $1 / t^{*}$ is an eigenvalue of $T\left(E^{*}\right)$. In particular, for real $E$, the $2 M$ eigenvalues of $T(E)$ occur in pairs $t, 1 / t^{*}$, unless $|t|=1$. The number $\nu$ of pairs of eigenvalues on the unit circle coincides with the number of bands with intersection in $E$ [18].

Let us denote the eigenvalues of $T(E), E$ real, as

$$
t_{a}=e^{ \pm \lambda_{a}+i \theta_{a}}, \quad a=1, \ldots, M-\nu, \quad t_{b}=e^{i \theta_{b}}, \quad b=1, \ldots, 2 \nu
$$


and write the modulus of (3.1), with real $E$ and $z=e^{i \varphi}$, in terms of them. After some simple algebra:

$$
\begin{gathered}
\prod_{a=1}^{M-\nu}\left(2 \cosh \lambda_{a}-2 \cos \left(\theta_{a}-\varphi\right)\right) \prod_{b=1}^{2 \nu} 2 \sin \left(\frac{1}{2}\left|\theta_{b}-\varphi\right|\right)= \\
=\left|\operatorname{det}\left(L_{N-1} \ldots L_{1}\right)\right|^{-1}\left|\operatorname{det}\left[E-\mathcal{H}\left(e^{i \varphi}\right)\right]\right|
\end{gathered}
$$

By taking the logarithm of it and integrating the phase $\varphi$ in $[0,2 \pi)$ one obtains a remarkably simple and interesting relation:

$$
\sum_{a=1}^{M-\nu} \lambda_{a}(E)=-\sum_{j=1}^{N-1} \log \left|\operatorname{det} L_{j}\right|+\frac{1}{2 \pi} \int_{0}^{2 \pi} d \varphi \log \left|\operatorname{det}\left[E-\mathcal{H}\left(e^{i \varphi}\right)\right]\right|
$$

This formula is exact, and is valid for a single matrix. In the large $N$ limit and in a statistical context, one would have the average behaviour $\left\langle\lambda_{a}\right\rangle=N \gamma_{a}$, where $\gamma_{a}$ is independent of $N$. The right hand side of (3.5) would be evaluated by means of the ensemble and $\varphi$-averaged density of eigenvalues of $\mathcal{H}\left(e^{i \varphi}\right)$. The equation would then provide a generalization to $M>1$ of the Thouless relation between the Lyapunov spectrum and the average eigenvalue density of the Hamiltonian ensemble [10].

If instead we take in (3.1) the derivative in the variable $z$, by using the property $d / d z \operatorname{det}(A+z I)=\operatorname{det}(A+z I) \operatorname{tr}(A+z I)^{-1}$, we obtain equation (1.9). Also the derivative in the right hand side can be computed, and gives the following final formula, where $\tilde{G}_{i j}$ are the blocks of size $M \times M$ that partition the resolvent $\tilde{G}(z, E)=[\mathcal{H}(z)-E]^{-1}$ :

$$
\operatorname{Tr}\left(\frac{1}{T(E)-z}\right)=-\frac{M}{z}-\operatorname{Tr} \tilde{G}_{1, N}+\frac{1}{z^{2}} \operatorname{Tr} \tilde{G}_{N, 1}
$$

The same formula follows from a stronger result, to be given in the next section.

\section{$\S 4$ The transfer matrix and the resolvent of $\mathcal{H}(z)$.}

A representation of the resolvent of the transfer matrix can be given in terms of the resolvent of the non-Hermitian matrix, $\tilde{G}(z, E)=[\mathcal{H}(z)-E]^{-1}$. Note that $\left[\tilde{G}(z, E)_{i j}\right]^{\dagger}=$ $\tilde{G}\left(1 / z^{*}, E^{*}\right)_{j i}$. With the same procedure as in $\S 2$, one obtains two identities

$$
\left(\begin{array}{c}
z \tilde{G}_{1,1} \\
\tilde{G}_{N, 1}
\end{array}\right)=T(E)\left(\begin{array}{c}
\tilde{G}_{1,1} \\
\frac{1}{z} \tilde{G}_{N, 1}-I
\end{array}\right), \quad\left(\begin{array}{c}
z \tilde{G}_{1, N}-I \\
\tilde{G}_{N, N}
\end{array}\right)=T(E)\left(\begin{array}{c}
\tilde{G}_{1, N} \\
\frac{1}{z} \tilde{G}_{N, N}
\end{array}\right)
$$

which join into the matrix relation

$$
\left(\begin{array}{cc}
z \tilde{G}_{1, N}-I & z \tilde{G}_{1,1} \\
\tilde{G}_{N, N} & \tilde{G}_{N, 1}
\end{array}\right)=T(E)\left(\begin{array}{cc}
\tilde{G}_{1, N} & \tilde{G}_{1,1} \\
\frac{1}{z} \tilde{G}_{N, N} & \frac{1}{z} \tilde{G}_{N, 1}-I
\end{array}\right)
$$

Simple steps lead to the final representation:

$$
\frac{1}{T(E)-z}=\left(\begin{array}{cc}
-\tilde{G}_{1, N} & \frac{1}{z} \tilde{G}_{1,1} \\
-\frac{1}{z} \tilde{G}_{N, N} & \frac{1}{z^{2}} \tilde{G}_{N, 1}-\frac{1}{z} I
\end{array}\right)
$$


which, by taking the trace, provides eq. (3.6).

Note that the corner blocks of $\tilde{G}$ can be expressed in closed form in terms of the corner blocks of $G$, by means of the Lippman Schwinger equations

$$
G_{i, j}=\tilde{G}_{i, j}+\frac{1}{z} G_{i, 1} \tilde{G}_{N, j}+z G_{i, N} \tilde{G}_{1, j}
$$

\section{$\S 5$ The matrix $T^{\dagger} T$.}

The general results obtained so far for transfer matrices can also be applied to the matrix $Q(E) \equiv T(E)^{\dagger} T(E)$, which will be shown to be itself the transfer matrix of a Hamiltonian built out of $\mathcal{H}$.

The matrix $Q(E)$ has the feature of being Hermitian and positive, therefore with real and positive eigenvalues. It is easy to show the validity of the property:

$$
Q\left(E^{*}\right) \sigma_{2} Q(E)=\sigma_{2}
$$

It follows that if $t$ is an eigenvalue of $Q(E)$, then $1 / t$ is eigenvalue of $Q\left(E^{*}\right)$.

While considering the factorization

$$
T(E)^{\dagger} T(E)=T_{1}(E)^{\dagger} \Sigma_{1}^{\dagger} T_{2}(E)^{\dagger} \ldots \Sigma_{N-1}^{\dagger} T_{N}(E)^{\dagger} T_{N}(E) \Sigma_{N-1} \ldots \Sigma_{1} T_{1}(E)
$$

one notes the property that $T_{k}(E)^{\dagger}$, constructed with $H_{k}$, coincides with $-T_{k}\left(-E^{*}\right)$ constructed with $-H_{k}$. This allows to interpret $Q(E)$ as the transfer matrix for the solution of the equation $\mathcal{K}(E) \Psi=0$, with matrix $\mathcal{K}(E)=$

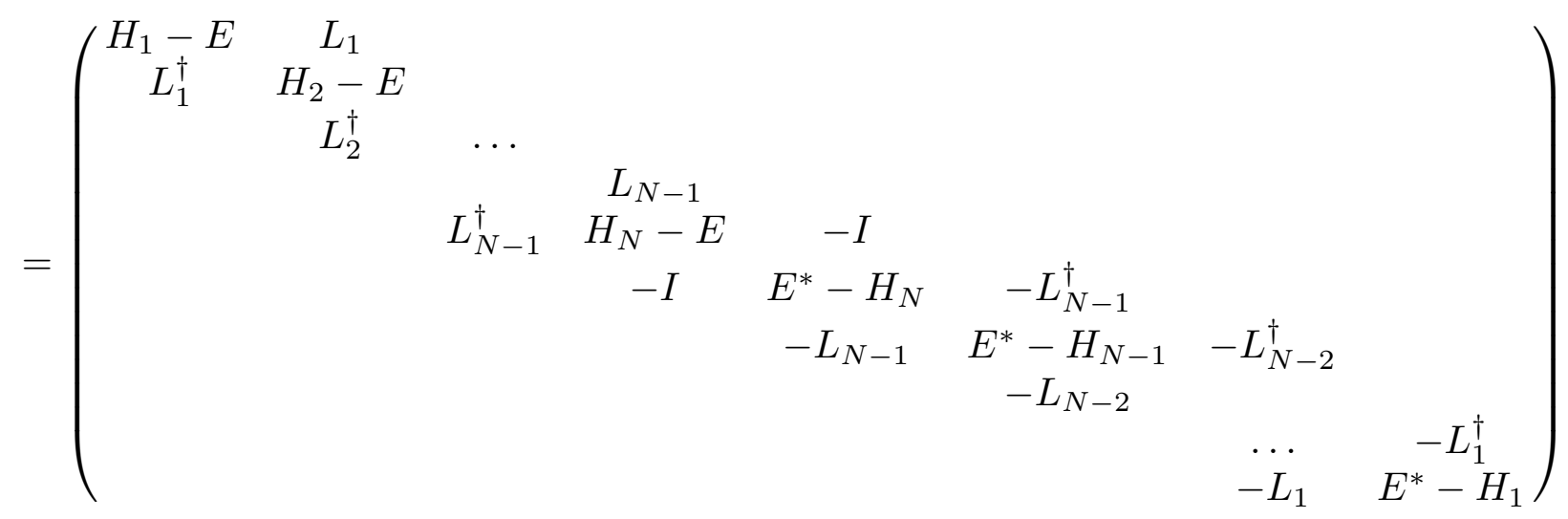

The corresponding non-Hermitian matrix $\mathcal{K}(E, z)$ entering the duality relation, is obtained by placing the diagonal matrices $z^{-1} I$ and $z I$ in the upper right and lower left corners respectively. Then, the following equation holds:

$$
\operatorname{det}\left[T(E)^{\dagger} T(E)-z\right]=(-1)^{N M}(-z)^{M}\left|\operatorname{det}\left(L_{N-1} \ldots L_{1}\right)\right|^{-2} \operatorname{det} \mathcal{K}(E, z)
$$

A few remarks on the spectral properties of $\mathcal{K}(E, z)$, which can be easily translated for the matrix $\mathcal{K}(E)$, are useful: 
a) $\mathcal{K}(E, z)^{\dagger}=\mathcal{K}\left(E^{*}, 1 / z^{*}\right)$;

b) $\left(\begin{array}{cc}0 & P \\ -P & 0\end{array}\right) \mathcal{K}(E, z)\left(\begin{array}{cc}0 & -P \\ P & 0\end{array}\right)=-\mathcal{K}\left(E^{*}, 1 / z\right)$

where $P$ is the block matrix with nonzero blocks $P_{i, N-i+1}=I$ of size $M, i=1 \ldots N$;

c) for real $z$, because of a) and b), the eigenvalues of $\mathcal{K}(E, z)$ come in pairs $x,-x^{*}$;

d) the value $x=0$ does not belong to the spectrum of $\mathcal{K}(E, z)$ if $z$ is not in the real positive axis, because the left side in (5.2) cannot vanish.

Let us concentrate on the case where $z=e^{i \varphi}$ and $E$ is real; the matrix $\mathcal{K}\left(E, e^{i \varphi}\right)$ is Hermitian, therefore its eigenvalues are real. By point c) the eigenvalues for $\varphi=0, \pi$ are symmetric; they also mark the extrema of the bands [18]: it follows that $\mathcal{K}\left(E, e^{i \varphi}\right)$ has as many positive as many negative eigenvalues and $(-1)^{N M} \operatorname{det} \mathcal{K}\left(E, e^{i \varphi}\right)>0$. The eigenvalues of $Q(E)$ are $M-\mu$ positive pairs $\left(t_{a}, t_{a}^{-1}\right)$, with $t_{a}=e^{\lambda_{a}}>1$, being $2 \mu$ eigenvalues equal to unity. Equation (5.2) reads, in terms of the eigenvalues of $Q(E)$ :

$$
\left(2 \sin \frac{\varphi}{2}\right)^{2 \mu} \prod_{a=1}^{M-\mu}\left(2 \cosh \lambda_{a}-2 \cos \varphi\right)=\prod_{k=1}^{N-1}\left|\operatorname{det} L_{k}\right|^{-2}(-1)^{N M} \operatorname{det} \mathcal{K}\left(E, e^{i \varphi}\right)
$$

By taking the logarithm and integrating in $\varphi$, we end with a formula of Thouless type:

$$
\sum_{a} \lambda_{a}(E)=-2 \sum_{k=1}^{N-1} \log \left|\operatorname{det} L_{k}\right|+\frac{1}{2 \pi} \int_{0}^{2 \pi} d \varphi \log \left[(-1)^{N M} \operatorname{det} \mathcal{K}\left(E, e^{i \varphi}\right)\right]
$$

For real $z$ and $E$ real or complex it is convenient, at least for a simplification of the notation, to bring the matrix $\mathcal{K}(E, z)$ to another form; there is much freedom since only the determinant of the matrix matters. Let us choose to left and right-multiply matrix $\mathcal{K}(E, z)$ by unitary matrices to give:

$$
\begin{aligned}
\mathcal{K}^{\prime}(E, z) & \equiv \frac{1}{\sqrt{2}}\left(\begin{array}{cc}
I & P \\
-i I & i P
\end{array}\right) \mathcal{K}(E, z) \frac{1}{\sqrt{2}}\left(\begin{array}{cc}
-I & i I \\
P & i P
\end{array}\right) \\
& =\left(\begin{array}{cc}
\mathcal{H}-\operatorname{Re} E+U & -i V-\operatorname{Im} E \\
i V-\operatorname{Im} E & -\mathcal{H}+\operatorname{Re} E+U
\end{array}\right)
\end{aligned}
$$

where $P$ is the same matrix of size $N M$ defined after (5.2), $U$ and $V$ are block diagonal matrices, each of the $N$ diagonal blocks having size $M$. The only nonzero blocks are: $U_{1,1}=\frac{1}{2}\left(z-z^{-1}\right) I, V_{1,1}=\frac{1}{2}\left(z+z^{-1}\right) I$ and $V_{N, N}=-I$. With this transformation, we obtain the equivalent form of the duality relation:

$$
\operatorname{det}\left[T(E)^{\dagger} T(E)-z\right]=(-1)^{N M}(-z)^{M}\left|\operatorname{det}\left(L_{N-1} \ldots L_{1}\right)\right|^{-2} \operatorname{det} \mathcal{K}^{\prime}(E, z)
$$

The matrix $\mathcal{K}^{\prime}(E, z)$ is Hermitian for any $E$ in the complex plane and real $z$, and it has the advantage of containing the matrix $\mathcal{H}$ in the diagonal blocks, albeit with opposite sign; a similar structure appears in a paper by Efetov [24]. It has the following properties:

a) $\mathcal{K}^{\prime}(E, z)^{\dagger}=\mathcal{K}^{\prime}\left(E, z^{*}\right)$ 
b) $\left(\begin{array}{ll}0 & I \\ I & 0\end{array}\right) \mathcal{K}^{\prime}(E, z)\left(\begin{array}{cc}0 & I \\ I & 0\end{array}\right)=-\mathcal{K}^{\prime}\left(E^{*}, 1 / z\right)$

c) $\left(\begin{array}{cc}I & 0 \\ 0 & -I\end{array}\right) \mathcal{K}^{\prime}(E, z)\left(\begin{array}{cc}I & 0 \\ 0 & -I\end{array}\right)=\mathcal{K}^{\prime}\left(E^{*},-1 / z\right)$

\section{$\S 6$ Conclusions.}

In the present paper and in [18] I have considered a class of Hamiltonians characterized by a block Jacobi structure which is shared in many interesting models of quantum disordered transport. For a single Hamiltonian matrix I have obtained exact relations that allow to describe spectral properties of transfer matrices through properties of the Hamiltonian itself. The identities involve general boundary conditions that imply a close connection between transfer matrices and Non-Hermitian Hamiltonians, and make the statistical analysis less involved, since the statistical ensemble is usually defined for the Hamiltonian.

Some equations, like (1.9), are suited for the supersymmetric technique. Two problems arise, that have already been considered in the literature [25]: 1) the need of a special formalism for the determination of the density of complex eigenvalues from the knowledge of the average resolvent, 2) the "Hermitianization" procedure for representing ratio of determinants, which provide by differentiation traces of resolvents, as Gaussian superintegrals. These problems are absent while considering the relation for $T^{\dagger} T$. 


\section{References.}

[1] B.Kramer and A.MacKinnon: "Localization: theory and experiment", Rep. Progr. Phys. 56 (1993) 1469.

[2] T.Dröse, M.Batsch, I.Zharekeshev and B.Kramer: "Phase diagram of localization in a magnetic field", Phys. Rev. B 57 (1998) 37.

[3] A.Eilmes, R.A.Römer and M.Schreiber: "The two-dimensional Anderson model of localization with random hopping" Eur. Phys. J. B 1 (1998) 29;

T.Kawarabayashi, B.Kramer and T.Ohtsuki: "Anderson transition in threedimensional disordered systems with randomly varying magnetic flux", Phys. Rev. B 57 (1998) 11842.

[4] Y.V.Fyodorov and A.D.Mirlin: "Scaling properties of localization in Random Band Matrices: a $\sigma$-model approach", Phys. Rev. Lett. 67 (1991) 2405;

P.G.Silvestrov: "Summing graphs for random band matrices", Phys. Rev. E 55 (1997) 6419 .

[5] G.Casati, B.V.Chirikov, I.Guarneri and F.M.Izrailev: "Quantum ergodicity and localization in conservative systems: the Wigner band random matrix model", Phys. Lett. A 223 (1996) 430.

[6] G.Casati, I.Guarneri, L.Molinari and K.Życzkowski: "Periodic Band Random matrices and conductance in disordered media", Phys. Rev. Lett. 72 (1994) 2697;

G.Casati, I.Guarneri and G.Maspero: "Landauer and Thouless conductance: a Band

Random Matrix approach", J. Phys. I France 7 (1997) 729;

S.Iida, A.Weidenmüller and J.A.Zuk: "Statistical scattering theory, the supersymmetric method and universal conductance fluctuations", Ann. of Phys. 200 (1990) 219.

[7] Ph. Jacquod and D.L.Shepelyansky: "Hidden Breit-Wigner distribution and other properties of random band matrices with preferential basis", Phys. Rev. Lett. 75 (1995) 3501.

[8] E.Brézin and A.Zee: "Lattices of matrices", Nucl. Phys. B 441 [FS] (1995) 409;

S.Hikami and A.Zee: "Complex random matrix models with possible applications to spin-impurity scattering in quantum Hall fluids", Nucl. Phys. B 446 [FS] (1995) 337;

E.Brézin, S.Hikami and A.Zee: "Oscillating density of states near zero energy for matrices made of blocks with possible application to the random flux problem", Nucl. Phys. B 464 [FS] (1996) 411.

[9] A.Crisanti, G.Paladin and A.Vulpiani: "Products of random matrices in statistical physics", Springer Series in Solid State Sciences vol. 104, Springer-Verlag 1993.

[10] D.J.Thouless: "A relation between the density of states and the range of localization for one-dimensional random systems", J. Phys. C: Solid State Phys. 5 (1972) 77.

[11] J.B.Pendry: "Symmetry and transport of waves in one-dimensional disordered systems", Advances in Physics 43 (1994) 461.

[12] M.Isopi and C,M.Newman: "The triangle law for Lyapunov exponents of large random matrices", Comm. Math. Phys. 143 (1992) 591.

[13] J.Cook and B.Derrida: "Lyapunov exponents of large, sparse random matrices and the problem of directed polymers with complex random weights", J. Stat. Phys. 61 (1990) 961. 
[14] C.W.Beenakker: "Random-matrix theory of quantum transport", Rev. Mod. Phys. 69 (1997) 731.

[15] A.MacKinnon and B. Kramer: "One parameter scaling of localization length and conductance in disordered systems", Phys. Rev. Lett. 47 (1981) 1546.

[16] P.Markos: "Universal scaling of Lyapunov exponents", J. Phys. A 30 (1997) 3441.

[17] T.Kottos, A.Politi, F.M.Izrailev and S.Ruffo: "Scaling properties of Lyapunov spectra for the band random matrix model", Phys. Rev. E 53 (1996) R5553;

T.Kottos, A.Politi and F.M.Izrailev: "Finite size corrections in Lyapunov spectra for Band Random Matrices", cond-mat/9801223.

[18] L.Molinari: "Transfer matrices and tridiagonal block Hamiltonians with periodic and scattering boundary conditions", J. Phys. A: Math. Gen. 30 (1997) 983.

[19] N.Hatano and D.R.Nelson: "Localization transition in quantum mechanics", Phys. Rev. Lett. 77 (1996) 570.

[20] I.Goldsheid and B.Khoruzhenko: "Distribution of eigenvalues in Non-Hermitian Anderson models", Phys. Rev. Lett. 80 (1998) 2897;

J.Feinberg and A.Zee: "Spectral curves of Non-Hermitean Hamiltonians", condmat/9710040.

[21] V.Gurarie and A.Zee: "Localization length from single-particle properties in disordered electronic systems", cond-mat/9802042.

N.M.Schnerb and D.R.Nelson: "Winding numbers, complex currents, and NonHermitian localization", Phys. Rev. Lett. 80 (1998) 5172.

[22] P.W.Brouwer, P.G.Silvestrov and C.W.J.Beenakker: "Theory of directed localization in one dimension", Phys. Rev. B 56 (1997) R4333.

[23] A.I.Aptekarev and E.M.Nikishin: "The scattering problem for a discrete SturmLiouville operator", Math. USSR Sbornik 49 (1984) 325.

[24] K.B.Efetov: "Quantum disordered systems with a direction", Phys. Rev. B 56 (1997) 9630.

[25] J.Feinberg and A.Zee: "Non-hermitian random matrix theory: method of hermitian reduction", Nucl. Phys. B 504 [FS] (1997) 579. 